\title{
Cardiorespiratory fitness in adolescents before and after the COVID-19 confinement: a prospective cohort study
}

\author{
Rubén López-Bueno ${ }^{1,2}$ (1) Joaquín Calatayud ${ }^{2,3}$ - Lars Louis Andersen ${ }^{2} \cdot$ José Casaña $^{3} \cdot$ Yasmín Ezzatvar $^{3}$. \\ José Antonio Casajús ${ }^{4}$. Guillermo Felipe López-Sánchez ${ }^{5} \cdot$ Lee Smith $^{6}$
}

Received: 25 February 2021 / Revised: 8 March 2021 / Accepted: 10 March 2021 / Published online: 17 March 2021

(C) The Author(s), under exclusive licence to Springer-Verlag GmbH Germany, part of Springer Nature 2021, corrected publication 2021

\begin{abstract}
Long periods of free-movement restrictions may negatively affect cardiorespiratory fitness and health. The present study investigated changes after the COVID-19 confinement in maximal oxygen intake $\left(\mathrm{VO}_{2}\right.$ max) levels in a sample of 89 Spanish school children aged 12 and 14 years at baseline ( $49.8 \%$ girls). The 20 - $\mathrm{m}$ shuttle run test served to estimate $\mathrm{VO}_{2}$ max before and after the COVID-19 confinement. Paired $t$-tests estimated an overall difference of $-0.5 \mathrm{ml} \cdot \mathrm{kg}^{-1} \cdot \mathrm{min}^{-1}$ (SD 0.3) $(p=0.12)$, whereas the highest significant reductions were observed for girls aged 14 years $\left(-1.5 \mathrm{ml} \cdot \mathrm{kg}^{-1} \cdot \mathrm{min}^{-1}\right.$ (SD 0.6) $(p<0.05)$ ). Boys aged 14 years showed a slight increase $\left(0.4 \mathrm{ml} \cdot \mathrm{kg}^{-1} \cdot \mathrm{min}^{-1}\right.$ (SD 0.5) $\left.(p=0.44)\right)$, whereas boys aged 12 years presented an important decrease $\left(-1.2 \mathrm{ml} \cdot \mathrm{kg}^{-1} \cdot \mathrm{min}^{-1}\right.$ (SD 0.7) $\left.(p=0.14)\right)$. Healthy Fitness Zone (HFZ) levels also experienced a decrease of $-3.4 \%$ as regards baseline levels over the examined period. All the examined subgroups showed lower levels in relation to a normal $\mathrm{VO}_{2}$ max rate development, although girls aged 14 and boys aged 12 years accounted for the highest part.

Conclusion: The results indicate that COVID-19 confinement might delay the normal development of $\mathrm{VO}_{2}$ max in adolescents. Strategies to tackle this concerning decline are warranted.
\end{abstract}

What is Known:

- First study analyzing cardiorespiratory fitness levels in teenagers after COVID-19 confinement.

What is New:

- Important delay in maximal oxygen intake identified in a sample of Spanish teenagers.

- These results should be considered to develop strategies of a more active lifestyle in teenagers during and after confinements.

Keywords Maximal oxygen peak $\cdot$ Children $\cdot$ Physical fitness $\cdot$ Growth $\cdot$ Lockdown

Communicated by Daniele De Luca

Rubén López-Bueno

rlopezbu@unizar.es

Joaquín Calatayud

ximo86@hotmail.com

Lars Louis Andersen

1la@nfa.dk

José Casaña

jose.casana@uv.es

Yasmín Ezzatvar

yasmin.ezzatvar@uv.es

José Antonio Casajús

joseant@unizar.es

Guillermo Felipe López-Sánchez

gfls@um.es
Lee Smith

Lee.Smith@aru.ac.uk

1 Department of Physical Medicine and Nursing, University of Zaragoza, Zaragoza, Spain

2 National Research Centre for the Working Environment, Copenhagen, Denmark

3 Exercise Intervention for Health Research Group (EXINH-RG), Department of Physiotherapy, University of Valencia, Valencia, Spain

4 Faculty of Health Sciences, University of Zaragoza, Zaragoza, Spain

5 Faculty of Sport Sciences, University of Murcia, Murcia, Spain

6 Cambridge Centre for Sport and Exercise Science, Anglia Ruskin University, Cambridge, UK 


\section{Introduction}

Adolescence is a critical period of human development characterized by profound physiological changes that lead to adulthood. The adherence to healthy habits is particularly relevant at this stage of life since those can importantly influence essential indicators linked to future health outcomes. Particularly, cardiorespiratory fitness is considered a critical hallmark for health during youth, and maximal oxygen uptake $\left(\mathrm{VO}_{2} \max \right)$ a good indicator for it [1]. Higher levels of $\mathrm{VO}_{2}$ max in childhood and adolescence have been associated with lower values of cardiovascular risk factors such as waist circumference, body mass index (BMI), body fatness, blood pressure, total cholesterol, high-density lipoprotein cholester$\mathrm{ol}$, triglycerides, and prevalence of metabolic syndrome in later life $[2,3]$. Despite this concern, recent research has estimated a $\mathrm{VO}_{2}$ max decline of $7.3 \%$ among children and adolescents from both high-income and upper middle-income countries over the last decades [4], which could be attributed to a reduction of physical activity during that period [5]. In fact, an increase in physical activity levels has been associated with higher $\mathrm{VO}_{2}$ max values in adolescents, especially with those previously inactive or overweight [6]. Therefore, it seems of upmost importance for adolescents to achieve sufficient levels of physical activity that can preserve reliable health indicators such as $\mathrm{VO}_{2}$ max.

Due to the unprecedented situation regarding the ongoing COVID-19 pandemic, there is no knowledge on how the restrictions imposed for enacted COVID-19 confinements might have influenced cardiorespiratory fitness levels among the population. Although it is known that prolonged periods of bed rest correspond to a gradual $\mathrm{VO}_{2}$ max drop of around $0.3-0.4 \% /$ day in young adults [7], it is quite unlikely that confined adolescents have spent most of their time completely inactive. Nevertheless, prior research has suggested that levels of physical activity among children and adolescents decreased in countries with strict free-movement restrictions such as Spain [8]. Thus, as a result of a decrease in their physical activity levels, it is quite plausible that cardiorespiratory fitness levels among youth might have been reduced over that period. While closure of schools was established worldwide during 2020 [9], such measures along with sport facility closures or the subsequent sudden halt of active commuting may have caused an increase in sedentary behaviours that subsequently led to $\mathrm{VO}_{2}$ max reductions in adolescents [10].

To date, there is no information on how $\mathrm{VO}_{2}$ max has evolved during these first months of the COVID-19 pandemic among adolescents, although it is reasonable to expect that enacted free-movement restrictions have led to meaningful decreases. The aim of the study was to evaluate cardiorespiratory fitness changes after the COVID-19 confinement among adolescents. Therefore, we hypothesized a reduction of levels of $\mathrm{VO}_{2}$ max among adolescents after COVID-19 confinement.

\section{Methods}

\section{Design and study population}

A prospective cohort study to assess $\mathrm{VO}_{2}$ max before and after COVID-19 confinement was conducted using a sample of school-aged adolescents residing in Spain. The enacted Spanish strict confinement due to COVID-19 comprised 6 consecutive weeks from 15 March 2020 to 24 April 2020 and implied full closure of schools and non-essential workplaces; that did not allow any free movement of minors outside households unless any medical reason or, for the case of those aged 15 or over, to do the shopping or take a dog for a single daily short walk. From then on, several stages of deescalation measures were implemented, starting with a relaxed confinement, in which minors aged below 14 years were permitted to go outside once a day for no more than an hour, accompanied by an adult, in a specific band time (from 25 April 2020 to 10 May 2020). After the phase of relaxed confinement, a progressive multi-stage process driven by regional governments was carried out; that combined different sets of measures involving movement restrictions among regional and municipal borders, limitations in sports clubs' practices and official tournaments, and restriction to access sports facilities among others, which continue in the present day [11].

The study sample collected data on $\mathrm{VO}_{2}$ max and BMI and included students aged 12 and 14 years at baseline from respectively two and three groups of 1 st and 3rd grades of secondary education from a North-East Spanish high school. The groups included in the study were selected through cluster randomization from among 12 potential groups comprising students of 1 st to 4 th secondary education grades. Data comprising both baseline (from 20 to 22 November 2019, 114 days before the start of the enacted Spanish strict confinement) and second assessment (from 18 to 20 November 2020, 192 days after the final of the relaxed confinement) were included in the study. Before the study enrolment, either parents or legal guardians were informed about the aim of the project, provided information about the current health status of their children through an online questionnaire, and signed an informed consent.

The study followed the principles of the World Medical Declaration of Helsinki, received the approval of the Ethics Committee of Research in Humans of the University of Valencia (register code 1510464), and adhered to the Strengthening the Reporting of Observational Studies in Epidemiology (STROBE) guidelines [12]. 


\section{Maximal oxygen uptake}

Levels of $\mathrm{VO}_{2}$ max were estimated through the original 1-min protocol of the 20-m shuttle run test, a continuous incremental multi-stage audio-guided field-based test [13], which has shown good reliability to predict $\mathrm{VO}_{2}$ max in children and adolescents when using the original equation $(r=0.71)$ (SD $5.9 \mathrm{ml} \cdot \mathrm{kg}^{-1} \cdot \mathrm{min}^{-1}$ ) [13]. Initial race speed was set to $8.5 \mathrm{~km} / \mathrm{h}$ at the beginning of the test, with a $0.5 \mathrm{~km} / \mathrm{h}$ increase every minute; each minute corresponded to a different test stage. The test was carried out in the same outdoor asphalt basketball court during the two assessment rounds (i.e., before and after COVID-19 confinement) in rounds comprised of 5 students alphabetically sorted within each of the five groups. Participants ran $20 \mathrm{~m}$ back and forth between two bounding lines, synchronizing their speed race with the pace set by the pre-recorded audio signals. Two researchers involved in the development of the tests registered the last stage and shuttle that each participant was able to complete. The test finished for each single participant when the bounding line was not stepped on time twice or when the participant voluntarily left the test. Verbal encouragement was standardized for the researchers with the following sentences: "Do your best" at the end of the initial instructions, and "Well done" which was provided each minute during the test. The weather conditions (relative humidity ranging from 60 to $70 \%$ and temperatures oscillating between 8 and $15^{\circ} \mathrm{C}$ ) and the time bands (from 10 a.m. to 1 p.m.) were similar for the two assessment rounds. During the second assessment round, a 2-m space among participants was marked on the surface to keep a safety distance. Participants were permitted to remove their facemasks only when performing the test. All of them had previously performed the test at least once before the first-round stage and were given the same instructions before the start.

To estimate levels of $\mathrm{VO}_{2}$ max, the Léger et al. [13] prediction equation was used:

$\mathrm{VO}_{2} \max \left(\mathrm{ml} \mathrm{kg}^{-1} \mathrm{~min}^{-1}\right)=31.025$

$$
\begin{aligned}
& +3.238 \text { speed }-3.248 \text { age } \\
& +0.1536 \text { speed } \times \text { age }
\end{aligned}
$$

where speed is the speed of the last completed stage $(\mathrm{km} / \mathrm{h})$ and age is the age at the last birthday.

Additionally, the Healthy Fitness Zone ${ }^{\circledR}$ (HFZ) charts (Cooper Institute, TX, USA) [14] in accordance with sex and age of each participant were used to classify them between those who met the considered healthy fitness levels or not in the two rounds of tests.

\section{Body mass index}

Weight and height were assessed on the day of performing the first assessment round, just before performing the test.
Participants were wearing either shorts or sports leggings, tshirts, and socks when the measurements were taken. A medical scale with a height rod (Detecto 400 Series, MO, USA) served to estimate weight $(\mathrm{kg})$ and height $(\mathrm{m})$ which were used to calculate BMI through the following equation:

$\mathrm{BMI}=\frac{\mathrm{kg}}{\mathrm{m}^{2}}$.

To ensure enough statistic power and meaningful subgroups, the BMI variable was later categorized into tertiles.

\section{Statistical analyses}

Statistical analyses were conducted through Stata version 16.1 (StataCorp, TX, USA). A priori analyses determined a minimum sample size of 34 participants $(\beta=80 \%, \alpha=5 \%, \delta=$ $0.5)$. The Shapiro-Wilk test was applied to check the normality of continuous variables. Paired $t$-tests were performed overall as well as for each age, sex, and BMI to examine $\mathrm{VO}_{2}$ max differences within each subgroup. Independent $t$ tests were performed to examine differences among subgroups within categories. Differences in relation to the prevalence of HFZ were evaluated by McNemar chi-squared tests, informing the frequencies and percentages, percentage differences, and $\chi^{2}$ values for each subgroup. Levels of significance were set at $p<0.05$. Additionally, the standardized effect size was calculated using Cohen's $d$, classified as small $(0.20)$, medium (0.50), and large (0.80). There were no missing values for participants included in the study.

\section{Results}

Of the initial 128 participants, those with any chronic condition or infectious disease during any assessment stage were discarded from the study $(n=30)$. Moreover, participants whose parents refused giving informed consent to participate $(n=9)$ were also discarded. Therefore, 89 participants $(70 \%$ of the initial sample) with 1-year follow-up were finally included in the study.

Table 1 shows the main features of the study sample. A total of 89 adolescents on average aged 13.3 years (SD 0.9), of whom 44 (49.4\%) were girls, participated in the study. The BMI mean of the sample was $23.6 \mathrm{~kg} / \mathrm{m}^{2}$ (SD 3.9). The overall average $\mathrm{VO}_{2}$ max before COVID-19 confinement was 46.2 $\mathrm{ml} . \mathrm{kg}^{-1} \cdot \mathrm{min}^{-1}$ (SD 0.6), whereas the average $\mathrm{VO}_{2}$ max after COVID-19 confinement was $45.7 \mathrm{ml} \cdot \mathrm{kg}^{-1} \cdot \mathrm{min}^{-1}$ (SD 0.7), with an estimated difference of $-0.5 \mathrm{ml} \cdot \mathrm{kg}^{-1} \cdot \mathrm{min}^{-1}$ (SD 0.3) $(p=0.12)$ between the two periods. Subgroup analyses estimated a significant $\mathrm{VO}_{2}$ max reduction for the subgroup of girls ($1.0 \mathrm{ml} \cdot \mathrm{kg}^{-1} \cdot \mathrm{min}^{-1}$ (SD 0.4) $(p<0.05)$ ). Particularly, it was girls aged 14 years who presented the main difference of $\mathrm{VO}_{2}$ max, showing a reduction of $-1.5 \mathrm{ml} \cdot \mathrm{kg}^{-1} \cdot \mathrm{min}^{-1}$ (SD 0.6). The rest 


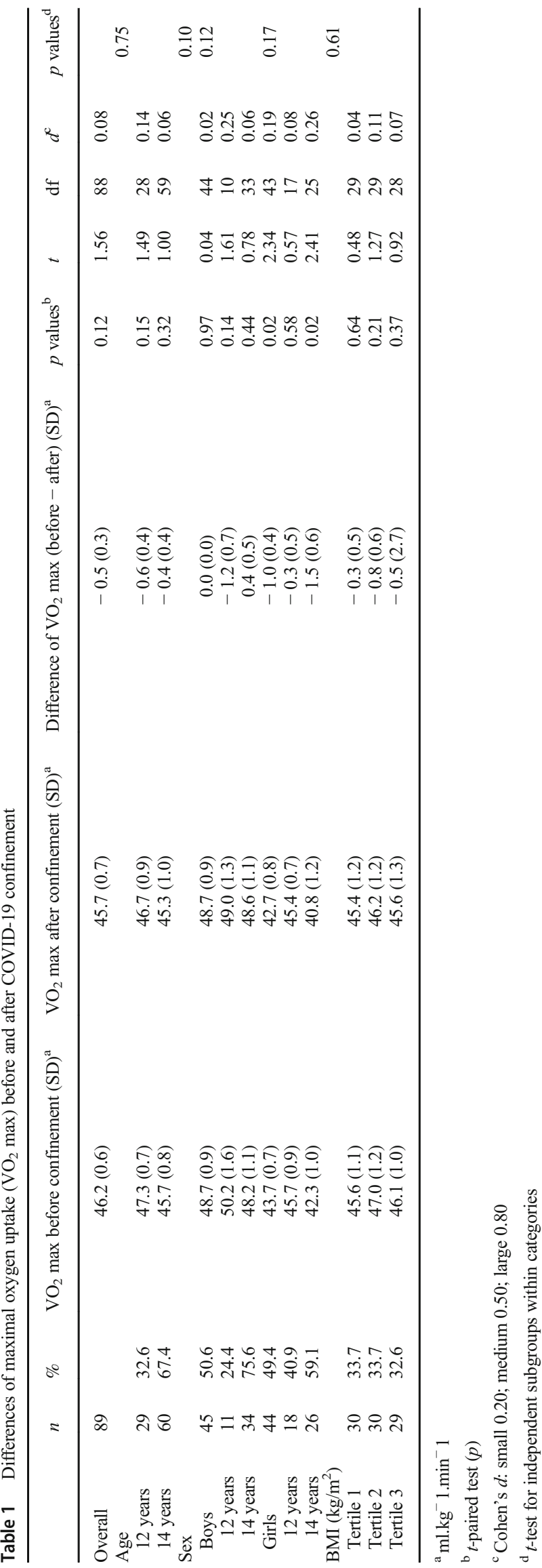

of the subgroups showed no differences or no significant reductions between the two examined periods except for boys aged 14 years, who showed no significant improvement of 0.4 ml. $\mathrm{kg}^{-1} \cdot \min ^{-1}(\mathrm{SD} 0.5)(p=0.44)$ in their $\mathrm{VO}_{2}$ max.

Regarding the prevalence of HFZ, Table 2 displays percentages of participants who met the recommended levels of HFZ. Overall, the prevalence of HFZ before and after COVID-19 confinement was respectively $79.8 \%$ and $76.4 \%$, with a lower percentage of participants meeting HFZ levels found for the second case $(-3.4 \%)\left(\chi^{2}=1.3\right)(p=0.26)$. Apart from boys aged 14 years $(3.0 \%)\left(\chi^{2}=1.0\right)(p=0.32)$, the rest of subgroups presented either no differences or lower percentages of HFZ after COVID-19 confinement. The highest reduction of HFZ was observed for girls aged 14 years ($15.4 \%)\left(\chi^{2}=2.7\right)(p=0.10)$, whereas BMI subgroups showed a dose-response fashion towards lower percentage of HFZ after COVID-19 confinement. All differences within subgroups were estimated as not statistically significant.

\section{Discussion}

The present study provides a new and original insight on how the COVID-19 confinement and other related enacted measures have affected $\mathrm{VO}_{2}$ max in a sample of Spanish adolescents. Until now, there is no study assessing $\mathrm{VO}_{2}$ max, a wellknown cardiorespiratory fitness indicator, and the potential detrimental effect of the COVID-19 confinement over it among adolescents. Contrary to expected, the reduction of $\mathrm{VO}_{2}$ max for the whole sample is small, and only specific subgroups such as girls and, particularly, girls aged 14 years presented statistically significant reductions. On the other hand, the subgroup of boys aged 14 years improved their $\mathrm{VO}_{2}$ max after the confinement. In addition, the percentage of participants meeting the recommended levels of HFZ was lower after the COVID-19 confinement, although such reduction represents a scarce percentage of the participants. Regarding BMI, no relevant differences were identified among or within subgroups, although all of them showed slight $\mathrm{VO}_{2}$ max reductions. Overall, our findings point at a lower impact than expected of the COVID-19 confinement over $\mathrm{VO}_{2}$ max of schooled teenagers. These findings might be owing to several reasons, comprising compensation strategies to improve health-related habits during the COVID-19 confinement, a profitable use of both the relaxed confinement and de-escalation phases concerning physical activity and physical development.

Despite that lower levels of physical activity among children and adolescents were observed to decrease in countries with stricter COVID-19 confinements [15, 16], levels of $\mathrm{VO}_{2}$ max in our study did not experience great variations. However, because $\mathrm{VO}_{2}$ max usually increases during adolescence due to the physical growth and development process per 
Table 2 Prevalence of Fitness Health Zone (FHZ) before and after COVID-19 confinement

\begin{tabular}{|c|c|c|c|c|c|c|c|c|c|}
\hline & \multicolumn{2}{|c|}{ FHZ before confinement } & \multicolumn{2}{|c|}{ FHZ after confinement } & \multirow{2}{*}{$\frac{\text { Difference of FHZ (before }- \text { after) }}{\%}$} & \multirow[t]{2}{*}{$\chi^{2}$} & \multirow[t]{2}{*}{ df } & \multirow[t]{2}{*}{$p$ value $^{\mathrm{a}}$} & \multirow[t]{2}{*}{$d^{b}$} \\
\hline & $n$ & $\%$ & $n$ & $\%$ & & & & & \\
\hline Overall & 71 & 79.8 & 68 & 76.4 & -3.4 & 1.3 & 1 & 0.26 & 0.08 \\
\hline \multicolumn{10}{|l|}{ Age } \\
\hline 12 years & 29 & 100 & 29 & 100.0 & 0.0 & 0.0 & 1 & 1.00 & 0.00 \\
\hline 14 years & 42 & 70.0 & 39 & 65.0 & -5.0 & 1.3 & 1 & 0.26 & 0.11 \\
\hline \multicolumn{10}{|l|}{ Sex } \\
\hline Boys & 36 & 80.0 & 37 & 82.2 & 2.2 & 1.0 & 1 & 0.32 & 0.06 \\
\hline 12 years & 11 & 100 & 11 & 100.0 & 0.0 & 0.0 & 1 & 1.00 & 0.00 \\
\hline 14 years & 25 & 73.5 & 26 & 76.5 & 3.0 & 1.0 & 1 & 0.32 & 0.07 \\
\hline Girls & 35 & 79.6 & 31 & 70.5 & -9.1 & 2.7 & 1 & 0.10 & 0.21 \\
\hline 12 years & 18 & 100 & 18 & 100.0 & 0.0 & 0.0 & 1 & 1.00 & 0.00 \\
\hline 14 years & 17 & 65.4 & 13 & 50.0 & -15.4 & 2.7 & 1 & 0.10 & 0.31 \\
\hline \multicolumn{10}{|l|}{ BMI $\left(\mathrm{kg} / \mathrm{m}^{2}\right)$} \\
\hline Tertile 1 & 22 & 73.3 & 24 & 80.0 & 6.7 & 2.0 & 1 & 0.16 & 0.16 \\
\hline Tertile 2 & 25 & 83.3 & 23 & 76.7 & -6.6 & 2.0 & 1 & 0.16 & 0.16 \\
\hline Tertile 3 & 24 & 82.3 & 21 & 72.4 & -9.9 & 3.0 & 1 & 0.08 & 0.25 \\
\hline
\end{tabular}

${ }^{a}$ McNemar's chi-squared test (FHZ before and after confinement)

${ }^{\mathrm{b}}$ Cohen's $d$ : small 0.20; medium 0.50; large 0.80

se, a deceleration of the $\mathrm{VO}_{2}$ max increase is still plausible; in normal children and adolescents, the $\mathrm{VO}_{2}$ max increases with growth and maturation, although girls usually reach their peak at around 14 years of age [17]. In general, a slight $\mathrm{VO}_{2} \max$ increase of $1 \mathrm{ml} \cdot \mathrm{kg}^{-1} \cdot \mathrm{min}^{-1}$ per year is expected in boys aged 12 to 15 years, whereas a decrease of around 0.5 $\mathrm{ml} \cdot \mathrm{kg}^{-1} \cdot \mathrm{min}^{-1}$ per year is considered normal among girls aged 12 to 18 years [14]. Thus, strictly in view of these mentioned rate developments of $\mathrm{VO}_{2}$ max, only girls aged 12 years have been able to maintain their expected $\mathrm{VO}_{2}$ max development over the examined period. Considering the limited duration of the strict confinement (i.e., 6 weeks) and the fact that there has been a relevant clearance period of several months from the end of both strict and relaxed confinements up to the second $\mathrm{VO}_{2}$ assessment (i.e., participants were permitted to do physical activity outdoors or moving among regional borders with specific limitations), the possibility that the detrimental effects of COVID-19 confinement over $\mathrm{VO}_{2}$ max have been mitigated during that time still exists. In fact, improvements of $\mathrm{VO}_{2}$ max owing to training have been commonly observed among adolescents [18]. Furthermore, a study by Stojmenović et al. [19] showed a lower improvement of $\mathrm{VO}_{2}$ max during adolescence in sedentary girls compared to trained girls; hence, the option of a high impact of the COVID-19 confinement on the $\mathrm{VO}_{2}$ max should not be discarded because that could have probably been mitigated by an ulterior increase of physical activity levels. By contrast, the effects of long COVID-19 (i.e., persisting symptoms in relation to previous SARSCoV-2 infection) worsening $\mathrm{VO}_{2}$ max are still plausible in children and adolescents since respiratory symptoms such as pain and chest tightness, fatigue, muscle, and joint pain have been previously observed even after 120 days of having been diagnosed, particularly among girls [20].

Moreover, since a compensation phenomenon regarding health-related behaviours has been observed during the COVID-19 confinement among adults [8], these types of behaviours concerning close family could have also influenced adolescents' levels of physical activity [21]. Additionally, the planning of active initiatives or the development of physically friendly environments might have positively influenced more active behaviours, especially during the relaxed confinement and the de-escalation phase [22-24]. Consequently, longer confinement periods, with less opportunities to do physical activity outdoors, might lead to more detrimental effects over $\mathrm{VO}_{2} \max$ than those observed in the present study. Also, the influence of warm weather during the de-escalation phase could have contributed to the increase levels of physical activity, since children, particularly girls, tend to be more physically active in such conditions [25]. Finally, even though BMI has been observed to affect levels of $\mathrm{VO}_{2}$ max in adolescents [26], our analyses did not identify any important pattern concerning this issue beyond the fact that it was participants from the second tertile who showcased higher $\mathrm{VO}_{2}$ max values in both assessments as well as the higher reductions. This could be partly explained by the interindividual variations of running economy observed among individuals with different or changing BMI [27]. Future research investigating both medium and long-term effects of strict confinements over $\mathrm{VO}_{2}$ max in adolescents is warranted, even more so that the cardiorespiratory fitness trends are showing an important decline over the last decades [4]. 


\section{Strengths and limitations}

The strengths of the current study comprise using a randomized sample of apparently healthy adolescents and the use of an objective cardiorespiratory fitness test, which has been validated to assess $\mathrm{VO}_{2}$ max. Another strength is that most of the eligible subjects chose to participate in the measurements. However, the findings of our study should be interpreted considering several limitations. The main constraint of the study is the inability to determine to what extent the observed results are related to COVID-19 confinement; the potential clearance period between the end of the strict and relaxed COVID-19 confinement and the second $\mathrm{VO}_{2}$ max assessment could have played its role in attenuating the detrimental effects. Besides, the 20-m shuttle run test is a field-based test that indirectly estimates $\mathrm{VO}_{2}$ max; thus, a potential information bias should not be discarded. Our study assumes that the $\mathrm{VO}_{2}$ max development rate from a large sample of American adolescents can be compared to Spanish ones. Nevertheless, average baseline levels of $\mathrm{VO}_{2}$ max of the present study are higher than their American counterparts [14] and could hamper both interpretations and generalizations. On the other hand, baseline $\mathrm{VO}_{2} \max$ levels estimated in this study are similar to those observed in prior research including a large representative sample of adolescents from the same Spanish region, which confers consistency to our $\mathrm{VO}_{2}$ estimations [28]. Also, because obese adolescents usually have lower $\mathrm{VO}_{2}$ max than their normal BMI counterparts, adolescents with higher levels of BMI might present different trends of $\mathrm{VO}_{2}$ max over the examined period [26]. Even though we drew a random sample from a specific region, we do not know if the present results can be generalized to all Spanish adolescents. Finally, examining other potential variables such as socioeconomic features might provide new perspectives on the research topic; however, since most of the participants from our study sample live in the same neighbourhood and study in the same high school, it is quite unlikely that a big effect size can be attributed to this. Also, because the present study did not include adolescents previously diagnosed with COVID-19, further research might also focus on adolescents that have experienced COVID-19 and how that has affected their $\mathrm{VO}_{2} \max$ over time.

Overall, the results suggest a delay in the expected evolution of $\mathrm{VO}_{2}$ max as regards to normal values during the examined period. Particularly boys aged 12 and girls aged 14 years showed important reductions in relation to what is expected for their age. The COVID-19 confinement has possibly affected the normal development of $\mathrm{VO}_{2}$ max in adolescents from Spain, who have experienced strict movement restrictions. Strategies promoting an active lifestyle to avoid deepening into the already declining trends of cardiorespiratory fitness in adolescents are warranted.
Abbreviations $\mathrm{VO}_{2}$ max, Maximal oxygen intake; BMI, Body mass index; SD, Standard deviation; HFZ, Healthy Fitness Zone

Authors' contributions RLB was responsible for the concept, design, data collection, and interpretation of data, drafted the initial manuscript, and approved the final manuscript. JC was responsible for the data collection and interpretation of data and approved the final manuscript. GFLS was responsible for the data collection and interpretation of data and approved the final manuscript. LLA was responsible for the interpretation of data and approved the final manuscript. JAC was responsible for the interpretation of data and approved the final manuscript. JC was responsible for the interpretation of data and approved the final manuscript. YE was responsible for the interpretation of data and approved the final manuscript. LS was responsible for the concept, design, data collection, interpretation of data, and data analysis, drafted the initial manuscript, and approved the final manuscript. All authors approved the final manuscript as submitted and agree to be accountable for all aspects of the work.

Data availability The data of the study are available upon reasonable request.

Code availability Not applicable.

\section{Declarations}

Ethics approval The study received the approval of the Ethics Committee of Research in Humans of the University of Valencia (register code 1510464).

Consent to participate All participants gave informed consent to participate.

Consent for publication All participants gave informed consent to publish data from the study.

Conflict of interest The authors declare no competing interests.

\section{References}

1. Raghuveer G, Hartz J, Lubans DR, Takken T, Wiltz JL, MietusSnyder M, Perak AM, Baker-Smith C, Pietris N, Edwards NM, On behalf of the American Heart Association Young Hearts Athero, Hypertension and Obesity in the Young Committee of the Council on Lifelong Congenital Heart Disease and Heart Health in the Young (2020) Cardiorespiratory fitness in youth: an important marker of health: a scientific statement from the American Heart Association. Circulation 142:E101-E118. https://doi.org/10.1161/ CIR.0000000000000866

2. Mintjens S, Menting MD, Daams JG, van Poppel MNM, Roseboom TJ, Gemke RJBJ (2018) Cardiorespiratory fitness in childhood and adolescence affects future cardiovascular risk factors: a systematic review of longitudinal studies. Sports Med 48: 2577-2605. https://doi.org/10.1007/s40279-018-0974-5

3. Hasselstrøm H, Hansen SE, Froberg K, Andersen LB (2002) Physical fitness and physical activity during adolescence as predictors of cardiovascular disease risk in young adulthood. Danish youth and sports study. An eight-year follow-up study. Int J Sports Med 23:27-31. https://doi.org/10.1055/s-2002-28458

4. Tomkinson GR, Lang JJ, Tremblay MS (2019) Temporal trends in the cardiorespiratory fitness of children and adolescents 
representing 19 high-income and upper middle-income countries between 1981 and 2014. Br J Sports Med 53:478-486. https://doi. org/10.1136/bjsports-2017-097982

5. Masanovic B, Gardasevic J, Marques A et al (2020) Trends in physical fitness among school-aged children and adolescents: A systematic review. Front Pediatr 8:627529. https://doi.org/10. 3389/fped.2020.627529

6. Nevill AM, Duncan MJ, Sandercock G (2020) Modeling the dose-response rate/associations between VO2max and selfreported Physical Activity Questionnaire in children and adolescents. J Sport Health Sci 9:90-95. https://doi.org/10.1016/j.jshs. 2019.05.001

7. Ried-Larsen M, Aarts HM, Joyner MJ (2017) Effects of strict prolonged bed rest on cardiorespiratory fitness: systematic review and meta-analysis. J Appl Physiol 123:790-799. https://doi.org/10. 1152/japplphysiol.00415.2017

8. López-Bueno R, Calatayud J, Casaña J, Casajús JA, Smith L, Tully MA, Andersen LL, López-Sánchez GF (2020) COVID-19 confinement and health risk behaviors in Spain. Front Psychol 11:1-10. https://doi.org/10.3389/fpsyg.2020.01426

9. Buonsenso D, Roland D, De Rose C, et al (2021) Schools closures during the COVID-19 pandemic. Pediatr Infect Dis J Publish Ah:15. https://doi.org/10.1097/INF.0000000000003052

10. Aires L, Pratt M, Lobelo F, Santos RM, Santos MP, Mota J (2011) Associations of cardiorespiratory fitness in children and adolescents with physical activity, active commuting to school, and screen time. J Phys Act Health 8(Suppl 2):198-205. https://doi.org/10.1123/ jpah.8.s2.s198

11. Aragón Regional Government (2020) Fases de la desescalada del confinamiento de mayo a septiembre de 2020. In: Desescalada del Confin. en Aragón. https://www.aragon.es/coronavirus/ desescalada-confinamiento. Accessed 15 Dec 2020

12. von Elm E, Altman DG, Egger M, Pocock SJ, Gøtzsche PC, Vandenbroucke JP, STROBE Initiative (2008) The Strengthening the Reporting of Observational Studies in Epidemiology (STROBE) statement: guidelines for reporting observational studies. J Clin Epidemiol 61:344-349. https://doi.org/10.1016/j. jclinepi.2007.11.008

13. Léger LA, Mercier D, Gadoury C, Lambert J (1988) The multistage 20 metre shuttle run test for aerobic fitness. J Sports Sci 6:93-101. https://doi.org/10.1080/02640418808729800

14. Eisenmann JC, Laurson KR, Welk GJ (2011) Aerobic fitness percentiles for U.S. adolescents. Am J Prev Med 41:106-110. https:// doi.org/10.1016/j.amepre.2011.07.005

15. Pietrobelli A, Pecoraro L, Ferruzzi A, Heo M, Faith M, Zoller T, Antoniazzi F, Piacentini G, Fearnbach SN, Heymsfield SB (2020) Effects of COVID-19 lockdown on lifestyle behaviors in children with obesity living in Verona, Italy: a longitudinal study. Obesity 28:1382-1385. https://doi.org/10.1002/oby.22861

16. López-Bueno R, López-Sánchez GF, Casajús JA, Calatayud J, GilSalmerón A, Grabovac I, Tully MA, Smith L (2020) Health-related behaviors among school-aged children and adolescents during the Spanish Covid-19 confinement. Front Pediatr 8:1-11. https://doi. org/10.3389/fped.2020.00573
17. Armstrong N, Welsman JR (1994) Assessment and interpretation of aerobic fitness in children and adolescents. Exerc Sport Sci Rev 22: 435-476. https://doi.org/10.1249/00003677-199401000-00016

18. Baxter-Jones ADG, Maffulli N (2003) Endurance in young athletes: it can be trained. Br J Sports Med 37:96-97. https://doi.org/ 10.1136/bjsm.37.2.96

19. Stojmenović T, Ćurčić D, Vukašinović-Vesić M et al (2018) Changes in maximal oxygen uptake during growth and development in girls who actively participate in basketball and non-athletes girls: a longitudinal study. Vojnosanit Pregl 75:481-486. https:// doi.org/10.2298/VSP150901326S

20. Buonsenso D, Munblit D, De Rose C, et al (2021) Preliminary evidence on long COVID in children. medRxiv 2021.01.23.21250375. https://doi.org/10.1101/2021.01.23. 21250375

21. Petersen TL, Møller LB, Brønd JC, Jepsen R, Grøntved A (2020) Association between parent and child physical activity: a systematic review. Int J Behav Nutr Phys Act 17:67. https://doi.org/10.1186/ s12966-020-00966-Z

22. Rhodes RE, Blanchard CM, Quinlan A, Naylor PJ, Warburton DER (2019) Family physical activity planning and child physical activity outcomes: a randomized trial. Am J Prev Med 57:135-144. https://doi.org/10.1016/j.amepre.2019.03.007

23. Pedroni C, Dujeu M, Moreau N, Lebacq T, Méroc E, Godin I, Castetbon K (2019) Environmental correlates of physical activity among children 10 to 13 years old in Wallonia (Belgium). BMC Public Health 19:1-12. https://doi.org/10.1186/s12889-019-6509-7

24. Maitland C, Stratton G, Foster S et al (2013) A place for play? The influence of the home physical environment on children's physical activity andsedentary behaviour. Int J Behav Nutr Phys Act 10:99. https://doi.org/10.1186/1479-5868-10-99

25. Rahman S, Maximova K, Carson V, Jhangri GS, Veugelers PJ (2019) Stay in or play out? The influence of weather conditions on physical activity of grade 5 children in Canada. Can J Public Health 110:169-177. https://doi.org/10.17269/s41997-019. 00176-6

26. Berndtsson G, Mattsson E, Marcus C, Larsson UE (2007) Age and gender differences in VO2max in Swedish obese children and adolescents. Acta Paediatr Int J Paediatr 96:567-571. https://doi.org/ 10.1111/j.1651-2227.2007.00139.x

27. Svedenhag J (1995) Maximal and submaximal oxygen uptake during running: how should body mass be accounted for? Scand J Med Sci Sports 5:175-180. https://doi.org/10.1111/j.1600-0838.1995. tb00033.x

28. Chillón P, Ortega FB, Ferrando JA, Casajus JA (2011) Physical fitness in rural and urban children and adolescents from Spain. J Sci Med Sport 14:417-423. https://doi.org/10.1016/j.jsams.2011. 04.004

Publisher's note Springer Nature remains neutral with regard to jurisdictional claims in published maps and institutional affiliations. 